\title{
Rapidly rotating spacetimes and collisional super-Penrose process
}

\author{
O. B. Zaslavskii \\ Department of Physics and Technology, \\ Kharkov V.N. Karazin National University, \\ 4 Svoboda Square, Kharkov 61022, Ukraine and \\ Institute of Mathematics and Mechanics, Kazan Federal University, \\ 18 Kremlyovskaya St., Kazan 420008, Russid*
}

\begin{abstract}
We consider generic axially symmetric rotating spacetimes and examine particle collisions in the ergoregion. The results are generic and agree with those obtained in the particular case of the rotating Teo wormhole in N. Tsukamoto and C. Bambi, Phys. Rev. D 91, 104040 (2015). It is shown that for sufficiently rapid rotation, the energy of a particle escaping to infinity can become arbitrary large (so-called superPenrose process). Moreover, this energy is typically much larger than the center-of mass energy of colliding particles. In this sense the situation differs radically from that for collisions near black holes.

PACS numbers: 04.70.Bw, 97.60.Lf
\end{abstract}

\section{INTRODUCTION}

Interest to high energy collisions of particles in the strong gravitational field significantly increased after the observation made in [1] (it also renewed interest to more early works on the closed subject [2] - [4]). It was shown there that if two particles collide near the extremal Kerr black hole, the energy in the centre of mass $E_{c . m}$. can grow unbound provided one of particles has fine-tuned parameters. This provoked a large series of works which extended this result to many other scenarios with unbounded $E_{c . m}$. Not going in details, all these scenarios can be classified in terms of three independent parameters. The first one is the proximity to the horizon (plus necessity of fine-tuning of one of particles). It is measured

\footnotetext{
*Electronic address: zaslav@ukr.net
} 
by the value of the lapse function $N$ in the point of collision. This is just the scenario of collisions suggested in [1] or its direct generalizations. Moreover, the actual horizon can be absent (say, a system is only on the threshold of its formation). The second relevant characteristics is the angular momentum $L$ of one of particles. If it is large and negative, $E_{c . m}$. can be also large. This is scenario of collisions inside the ergoregion, not necessarily close to the horizon (such a scenario was suggested in [5] and generalized in [6]).

Quite recently, a new type of scenario was considered in [7]. The authors considered particle collisions near the throat of the Teo wormhole [8]. It turned out that for sufficiently small throat areal radius, $E_{c . m}$. can be large. One can ask, what is the underlying reason of high $E_{c . m}$. for this problem and whether the results are due to specific features of the wormhole model or have a general character. The answer is found in [9] where it was shown that the effect of unbound $E_{c . m}$. stems from high values of the parameter $\omega$ responsible for rotation taken in the point of collision. In this sense, the effect is universal and applies to rapidly rotating wormholes and, in principle, to relativistic stars as well. (Although its astrophysical relevance remains unclear.) Thus we obtain the third option of getting unbound $E_{c . m .}$.

Up to now, we discussed the sources of high $E_{c . m}$. Meanwhile, there is one more aspect - the properties of debris that reach flat infinity after collision. Their energy $E$ is related to $E_{c . m}$. in a rather indirect way. One can ask - whether or not the efficiency of the energy extraction to infinity may also become unbound? (Following [10], we will use the term "super-Penrose process".) We can exploit the same classification in terms of three parameters $(N, L, \omega)$. (As is already known, there exists a counterpart to the high energy collisions that arises due to an electric charge [11] instead of rotation but we do not discuss this issue here. We also do not consider the influence of the electromagnetic field on the energy extraction that can, in principle, increase efficiency of the Penrose process [12]). As far as small $N$ is concerned, it turned out that for collisions near black holes, amplification of the energy is restricted [13] - [15] if both initial paricles moved along ingoing trajectories. If one of particles is on an outgoing trajectory, the energy $E$ can be quite large [16], [17] or even formally unbound [10], 18] but in the latter case very special conditions are required and it is hard to satisfy them [19], [20]. Due to recent results [21], the super-Penrose process is possible for the case of naked singularity in collisions where also small $N$ in the point of collision is required. Another possibility of the super-Penrose process, that arises due to large negative $L$ of one 
of particles was found in [22].

In the present work, we examine the third remaining possibility and consider collisions with large $\omega$. We will see that this can be considered as a potential source of the superPenrose process and has an universal character. We do not pretend to any astrophysical applications (that is another separate issue) and only demonstrate that this version of the super-Penrose process is possible in principle. In the particular case of the Teo wormhole [8], the results for the collisional Penrose process [23] are reproduced.

\section{BASIC EQUATIONS}

We consider the metric of the form

$$
d s^{2}=-N^{2} d t^{2}+g_{\phi}(d \phi-\omega d t)^{2}+\frac{d r^{2}}{A}+g_{\theta} d \theta^{2} .
$$

We restrict ourselves to the motion in the equatorial plane $\theta=\frac{\pi}{2}$. Then, we can always redefine the radial coordinate in such a way that $N^{2}=A$. It is implied that the metric coefficients do not depend on $t$ and $\phi$. Correspondingly, the Killing energy $E_{0}=-m u_{0}$ and the angular momentum $L=m u_{\phi}$ are conserved. Here, $m$ is the particle's mass, $\frac{d x^{\mu}}{d \tau}$ is the four-velocity, $\tau$ is the proper time. We assume that there is no horizon, so $N$ vanishes nowhere.

The equations of motion along the geodesics read

$$
\begin{gathered}
m \dot{t}=\frac{X}{N^{2}}, \\
m \dot{\phi}=\frac{L}{g_{\phi}}+\frac{\omega X}{N^{2}}, \\
X=E-\omega L,
\end{gathered}
$$

where dot denotes the derivative with respect to $\tau$. From the normalization condition

$$
u_{\mu} u^{\mu}=-1
$$

we obtain that the radial momentum

$$
m \dot{r}=\sigma Z,
$$

where

$$
Z=\sqrt{X^{2}-N^{2}\left(m^{2}+\frac{L^{2}}{g_{\phi}}\right)}
$$


$\sigma= \pm 1$ depending on the direction of motion. The forward-in-time condition $\dot{t}>0$ entails

$$
X>0
$$

\section{CENTRE OF MASS ENERGY FOR HEAD-ON COLLISIONS}

Let particles 1 and 2 collide. One can define the centre of mass energy $E_{c . m}$ according to

$$
E_{c . m .}^{2}=-P_{\mu} P^{\mu}
$$

where $P^{\mu}=m_{1} u_{1}^{\mu}+m_{2} u_{2}^{\mu}$ is the total momentum in the point of collision, subscripts label particles. Then,

$$
E_{c . m .}^{2}=m_{1}^{2}+m_{2}^{2}+2 m_{1} m_{2} \gamma
$$

where $\gamma=-u_{1 \mu} u_{2}^{\mu}$ is the Lorentz factor of relative motion.

For simplicity, we assume now that

$$
\begin{gathered}
L_{1}=L_{2}<0, \\
m_{1}=m_{2}=m, E_{1}=E_{2} \geq m \geq 0, \\
m_{3}=m_{4}=\mu .
\end{gathered}
$$

For the definiteness, let us consider head-on collision, so $\sigma_{1}=-\sigma_{2}$. Then, it follows from (2) - (6) that

$$
\gamma m^{2}=\frac{X_{1}^{2}+Z_{1}^{2}}{N^{2}}-\frac{L_{1}^{2}}{g_{\phi}}=\frac{2 \beta}{N^{2}}-m^{2},
$$

where

$$
\beta=X_{1}^{2}-\frac{N^{2} L_{1}^{2}}{g_{\phi}} .
$$

It is seen that

$$
\begin{gathered}
\beta \geq \frac{m^{2}}{2} N^{2} \geq 0 \\
E_{c . m .}^{2}=\frac{4 \beta}{N^{2}}
\end{gathered}
$$

Obviously,

$$
E_{c . m .} \geq 2 \mu .
$$

Then, using (17) we obtain the bound

$$
\mu^{2} \leq \frac{\beta}{N^{2}}
$$




\section{CONSERVATION LAWS IN THE POINT OF COLLISION}

The conservation of the energy and angular momentum in the collision gives us

$$
\begin{aligned}
& E_{1}+E_{2}=E_{3}+E_{4} \\
& L_{1}+L_{2}=L_{3}+L_{4} .
\end{aligned}
$$

As a consequence, we have

$$
X_{1}+X_{2}=X_{3}+X_{4}
$$

The conservation of the radial momentum reads

$$
Z \equiv \sigma_{1} Z_{1}+\sigma_{2} Z_{2}=\sigma_{3} Z_{3}+\sigma_{4} Z_{4}
$$

Condition (8) for particle 3 gives us $L_{3} \leq \frac{E_{3}}{\omega}$. For particle 4 with the conservation laws (20), (21) taken into account, it follows from the same condition that

$$
L_{3} \geq \frac{E_{3}-X_{1}-X_{2}}{\omega} .
$$

\section{SCENARIO 1: IDENTICAL PARTICLES WITH NONZERO ANGULAR MOMENTA}

In what follows, we assume that $\sigma_{3}=+1$ (particle 3 moves to infinity) and, similarily to [23], examine this process for two identical particles assuming (11) - (13) .

We obtain from (24) that

$$
\frac{E_{3}-2 E_{1}}{\omega}+2 L_{1} \leq L_{3} \leq \frac{E_{3}}{\omega} .
$$

Let us consider the collision with $\sigma_{1}=-\sigma_{2}$. Then, as particles are identical, the total radial momentum is equal to zero and $\sigma_{4}=-\sigma_{3}=-1$. Thus we have

$$
X_{3}^{2}-N^{2}\left(\mu^{2}+\frac{L_{3}^{2}}{g_{\phi}}\right)=X_{4}^{2}-N^{2}\left(\mu^{2}+\frac{L_{4}^{2}}{g_{\phi}}\right) .
$$

Equations (21), (20) now read

$$
\begin{aligned}
& 2 L_{1}=L_{3}+L_{4}, \\
& 2 E_{1}=E_{3}+E_{4} .
\end{aligned}
$$


We want to obtain large positive $E_{3}$, so $E_{4}$ should be large negative. This is possible in the ergoregion only, so we take

$$
g_{00}=-N^{2}+\omega^{2} g_{\phi}>0 .
$$

After some algebra, one finds from (26), (27), (28) that

$$
\begin{aligned}
& L_{3}=\frac{P}{Q}, \\
& X_{3}=\frac{K}{Q},
\end{aligned}
$$

where

$$
\begin{gathered}
P=X_{1} E_{3}-\beta, Q=\omega E_{1}-\frac{L_{1} g_{00}}{g_{\phi}} . \\
K=E_{3} L_{1} \frac{N^{2}}{g_{\phi}}+\omega \beta .
\end{gathered}
$$

Here,

$$
Q>0, E_{1}>0, L_{1}<0, g_{00}>0
$$

One can show that

$$
K>0
$$

so indeed $X_{3}>0$ in agreement with the forward-in-time condition. (The proof of this important property is done in Appendix.)

The condition $Z_{3}^{2} \geq 0$ gives us

$$
X_{3}^{2} \geq N^{2}\left(\mu^{2}+\frac{L_{3}^{2}}{g_{\phi}}\right) .
$$

By plugging (30), (32) into (36) we obtain the inequality for $E_{3}$ :

$$
E_{3}^{2}-2 E_{3} E_{1}-\beta \frac{g_{\phi}\left(\omega^{2}-\frac{N^{2}}{g_{\phi}}\right)}{N^{2}}+\frac{g_{\phi} Q^{2}}{\beta} \mu^{2} \leq 0 .
$$

Its solution turns out to be rather simple:

$$
\begin{gathered}
E_{ \pm}=E_{1} \pm s \\
s=\frac{Q \sqrt{g_{\phi}}}{N} \sqrt{B}, B=1-\frac{\mu^{2} N^{2}}{\beta} \\
E_{-} \leq E_{3} \leq E_{+} .
\end{gathered}
$$

As the Lorentz factor $\gamma>0$, it is seen from (19) that $B \geq 0$. 
The energy extraction is measured by the characteristics

$$
\eta=\frac{E_{3}}{E_{1}+E_{2}} .
$$

For the maximum possible energy $E_{+}$,

$$
\eta_{+}=\frac{1}{2}+\frac{\sqrt{B} \sqrt{g_{\phi}}}{2 N} \frac{Q}{E_{1}}=\frac{1}{2}+\frac{\sqrt{B} \sqrt{g_{\phi}}}{2 N}\left(\omega+\frac{\left|L_{1}\right| g_{00}}{E_{1} g_{\phi}}\right) .
$$

In the above formulas, all quantities are taken in the point of collision $r=\rho$. In partiuclar, $\omega=\omega(\rho)$.

\section{A. Rapid rotation}

Let us change parameters of the metric in such a way that, formally,

$$
\omega \rightarrow \infty
$$

in the point of collision. This is not to be confused with the behavior at inifnity since, in principle, this is consistent with the condition

$$
\lim _{r \rightarrow \infty} \omega=0
$$

for given values of parameters. A particular example from which this consistency can be seen is the Teo wormhole (see below in Sec. IX).

To avoid subtleties connected with the asymptotically nonflat spacetimes, in what follows we will assume that (44) is valid, so the energy at infinity has a clear and unambiguous meaning. Meanwhile, generalization to the metric that is not flat at infinity is also possible.

Then, in (29), it is the second term that dominates, provided that $g_{\phi}$ does not tend to zero too rapidly, so in the point of collision

$$
\begin{gathered}
\omega^{2} g_{\phi} \gg N^{2}, \\
g_{00} \approx \omega^{2} g_{\phi} \rightarrow \infty, \\
\beta \approx \omega^{2} L_{1}^{2}, \\
E_{+} \approx \frac{\omega^{2}\left|L_{1}\right| \sqrt{g_{\phi}}}{N} \sqrt{B},
\end{gathered}
$$




$$
\begin{aligned}
& X_{1}=E_{1}+\omega\left|L_{1}\right| \rightarrow \infty, \\
& L_{3} \leq L_{3}\left(E_{+}\right) \approx \omega\left|L_{1}\right| \frac{\sqrt{g_{\phi}}}{N} \sqrt{B} .
\end{aligned}
$$

Thus the extraction can become unbounded (as long as backreaction of particles on the metric is neglected), $\frac{E_{+}}{E_{1}} \gg 1$ when

$$
\omega^{2} \gg \frac{E_{1} N}{\left|L_{1}\right| \sqrt{g_{\phi}} \sqrt{B}} .
$$

Then, it follows from (15), (17) that

$$
E_{c . m .} \approx \frac{2 \omega\left|L_{1}\right|}{N}
$$

So

$$
\frac{E_{+}}{E_{c . m .}} \approx \frac{\sqrt{g_{\phi}} \sqrt{B} \omega}{2} \gg 1
$$

due to (45), (46).

In contrast to the situation with collisions near black holes where, as a rule, $E_{+}=O(1)$ and either $E_{c . m .}=O(1)$ or $E_{c . m}$. is unbound, here the situaiton is opposite: $E_{+}$becomes much larger than $E_{c . m .}$ !

\section{SCENARIO 2: IDENTICAL PARTICLES WITH ZERO ANGULAR MOMENTA}

It is seen from the previous formulas describing asymptotic behavior of the energies for large $\omega$ that there is a special case that deserves separate treatment. Let

$$
L_{1}=0=L_{2}
$$

Then, the asymtotic evaluaitons from the previous section do not apply but general formulas remain valid and are simplified. We have from (44), (7), (15), (19), (30), (32), (39) that

$$
\begin{gathered}
Z^{2}=E^{2}-N^{2} m^{2}, X=E, \beta=E_{1}^{2}, \\
\mu^{2} \leq \frac{E_{1}^{2}}{N^{2}} \\
L_{3}=\frac{E_{3}-E_{1}}{\omega}, \\
s=\frac{\omega E_{1} \sqrt{g_{\phi}}}{N} \sqrt{B}, B=1-\frac{\mu^{2} N^{2}}{E_{1}^{2}}
\end{gathered}
$$




$$
\begin{gathered}
E_{ \pm}=E_{1} \pm \frac{\omega \sqrt{g_{\phi}}}{N} \sqrt{E_{1}^{2}-\mu^{2} N^{2}}, \\
\eta_{+}=\frac{1}{2}+\frac{\omega \sqrt{g_{\phi}}}{2 N} \sqrt{1-\frac{\mu^{2} N^{2}}{E_{1}^{2}}} .
\end{gathered}
$$

In doing so, it follows from (17), (154) that

$$
E_{c . m .}^{2}=\frac{4 E_{1}^{2}}{N^{2}} .
$$

It is seen from (58), (60) that in the limit $\omega \rightarrow \infty$ the energy $E_{+}$grows but $E_{c . m}$. remains finite.

Thus the ratio

$$
\frac{E_{+}}{E_{c . m .}} \rightarrow \infty
$$

for both types of scenarios considered above (with $L_{1} \neq 0$ and $L_{1}=0$ ). If $g_{\phi}$ remains finite,

$\frac{E_{+}}{E_{c . m .}}=O(\omega)$ for both types of scenarios. However, if $g_{\phi} \rightarrow 0$ in this limit, the growth of this ratio is more slow. Such a situation happens just in the case of the Teo wormhole [8] where on the throat $\omega=O\left(b^{-3}\right)$ and $g_{\phi}=O\left(b^{2}\right)$ [7], [23] (see also below).

For the scenario under discussion, the mass of the escaping particle is restricted according to (55). There is no similar restriction for the previous type of scenario since the right hand side of (19) grows as $\omega^{2}$ for large $\omega$ according to (47).

\section{CAUSALITY, RAPID ROTATION AND THE ROLE OF ERGOREGION}

In our previous consideration the parameter $\omega$ is sent formally to infinity or, at least, becomes very large. On the first glance, this causes violation of causality since in the metric (1) the term $g_{\phi}(\omega d t)^{2}$ seems to dominate. Then, the interval apparently becomes spacelike that is impossible for any massive particle traveling with a speed less than a speed of light. Had this "obvious" conclusion been correct, this would have made all the above results as well as those of the previous works [7], [23] on this subject physically meaningless. Fortunately, this is not so.

First of all, one of essential ingredients in derivation of formulas is the normalization condition (5) that clearly tells us that a world line of particle is timelike, as it should be. But the question remains because of seeming contradiction described in the previous paragraph. To explain the issue under discussion, one is led to take into account the nature 
of a region in which we discuss our scenarios, i.e. the properties of the ergoregion. Outside it, there exist trajectories on which a particle can remain at rest. Then, one can neglect $d \phi$ in (1), obtain the spacelike interval and say that the limiting transition $\omega \rightarrow \infty$ is not legitimate.

However, inside the ergosphere the situation is qualitatively different. Meanwhile, it is the region (29) that we are interested in. It is the requirement of the timelike nature of a world line that makes a particle to rotate. It follows from (1) and the condition $d s^{2}<0$ that (say, for a circle orbit $r=$ const)

$$
\omega-\frac{N}{\sqrt{g_{\phi}}}<\frac{d \phi}{d t}<\omega+\frac{N}{\sqrt{g_{\phi}}} .
$$

On the border of the ergoregion (29), the left hand side vanishes, so inside this region a particle must rotate. Eq. (62) is well known and can be found in textbooks. We listed it here just because of our context in which we discuss the limiting transition $\omega \rightarrow \infty$. If eq. (62) is satisfied, one can take this limiting transition safely.

It is also instructive to look at the dynamic characteristics for the situation in question. According to eq. (15) of ref. [30], there exist he relation between the energy and angular momentum of a particle

$$
X=E-\omega L=\frac{m N}{\sqrt{1-V^{2}}}
$$

Here, $V$ is the velocity measured locally by the ZAMO (zero angular momentum observer [31]). The left hand side of (63) should be positive in view of (8) . Then, if $L<0$ and $N=O(1)$, we can take the limit $\omega \rightarrow \infty$. In doing so,

$$
V \approx 1-\frac{m^{2} N^{2}}{2 \omega^{2} L^{2}}
$$

so $V \rightarrow 1$ approaches the speed of light from below. Thus simultaneously $\frac{d \phi}{d t} \approx \omega \operatorname{can}$ be indefinitely large but, at the same time, $V$ remains slightly less than 1 , so causality is respected.

\section{RAPID ROTATION: WORMHOLES VERSUS BLACK HOLES}

It is instructive to compare the possibility of fast rotation for black holes and wormholes. Then, the counterpart of $\omega_{t h r}$ (subscript "thr" denotes quantities calculated on the throat 
of a wormhole) is the angular velocity of the horizon $\omega_{H}$. For the Kerr metric,

$$
\begin{gathered}
\omega_{H}=\frac{a}{2 M r_{+}}, \\
R=2 M,
\end{gathered}
$$

where $M$ is the black hole mass, $a=\frac{J}{M} \leq M, J \leq M^{2}$ is the angular momentum, the horizon radius $r_{+}=M+\sqrt{M^{2}-a^{2}}, R$ is the quantity of $\sqrt{g_{\phi \phi}}$ on the horizon in the equatorial plane $\theta=\frac{\pi}{2}$. One can construct the quantity

$$
v_{e}=R \omega_{H}
$$

that can be considered as a counterpart of the corresonding quantity for wormholes introduced in eq. (7) of [26].

Then, $v_{e}=\frac{a}{r_{+}}$, so the equation

$$
v_{e} \leq 1
$$

is satisfied.

In [26], where rotating wormholes supported by a phantom scalar field were considered, it was assumed that

$$
v_{e} \equiv \omega_{t h r} R \leq 1
$$

is satisfied, where now $R=\left(\sqrt{g_{\phi \phi}}\right)_{t h r}$.

Meanwhile, there is a big difference between both cases. For wormholes of Ref. [26], the mass-angular momentum relation reads

$$
M=2 \omega_{t h r} J
$$

Formally, it looks like the Smarr mass formula for the case of extremal black holes [32], if $\omega_{t h r}$ is replaced with $\omega_{H}$. But for such black holes $J=M^{2}$, so we obtain that $\omega_{H}=\frac{1}{2 M}$ in agreement with (65). Then, $v_{e}=1$. Thus for a given mass $M$ both $J$ and $\omega_{H}$ are fixed. Meanwhile, for the wormhole [26] it follows from (70) that $\omega_{t h r}=\frac{M}{2 J}$, where for a given $M$ we have freedom in changing $J$

If a black hole is nonextremal,

$$
J<M^{2}
$$

so in (67) $v_{e}<1$. 
The relevance of the inequality $J \leq M^{2}$ follows from the existence of a black hole horizon. But for wormholes there is no horizon and there is no such a restriction. Correspondingly, one should consider $M, J$ and $\omega_{t h r}$ as three parameters restricted by one relation (170) only, so we can take, say, $\omega_{t h r}$ as a free parameter. And, we can send it formally to infinity, without restriction (69).

Then, the quantity $v_{e} \rightarrow \infty$. It is certain that it has nothing to do with superluminal speed and causality violation. Outside the ergoregion, the quantity $v_{e}$ could be interpreted as a linear velocity of a particle with $r=$ const with respect to an observer at rest. But the throat for a big $\omega$ is located inside the ergosphere. In that region, such interpretation is impossible for the reasons explained above. Any observer rotates there. The simplest choice of an observer is the ZAMO one. Then, he is himself comoving with respect to this rapidly rotating throat. Instead, one may ask about the velocity of any geodesic particle with respect to the ZAMO frame. But, according to (64), it does not exceed the speed of light.

Thus the quantity $v_{e}$ inside the ergoregion does not have direct physical meaning. Moreover, this is so even in the black hole context. For example, although for extremal black holes $v_{e}=1$, as explained above, in the ZAMO frame the velocity of a particle on a marginally bound near-horizon orbit around the near-extremal Kerr hole has $V=1 / \sqrt{2} \neq v_{e}$ - see page 356 of [31] and generalization in [33].

In our context, fast rotation implies the validity of eq. (45). Taking $N=O(1)$, we obtain for wormholes of [26] the condition $\omega_{t h r} R \gg 1$ or $J \ll M R$. In this sense, for rapid rotation the angular momentum should be small. This unusual behavior can be attributed to the fact that the solutions considered in [26] are necessarily rotating. For a finite $\omega_{t h r}$ and $J \rightarrow 0$ the mass $M \rightarrow 0$ as well. If, instead, we want to keep $M$ nonzero even for small $J$, we are led to compensate the lack of angular momentum by increasing $\omega_{t h r}$. Further discussion of properties of the solutions [26] is beyond the scope of the present paper.

\section{COMPARISON WITH THE RESULTS FOR THE TEO WORMHOLE}

The scenarios with collisions of identical particles considered above are quite general in that the results apply to any metrric of the form (11). It is instructive to trace how the

particular results for the Teo wormhole [23] can be reproduced. For this wormhole [8] the 
metric coefficients in the equatorial plane read

$$
N=1, \omega=\omega(r, a)=\frac{2 a}{r^{3}}, g_{\phi}=r^{2} .
$$

Then, our eqs. (30) for the angular momentum with (32) taken into account, turns into eq. (4.22) of [23] where one should put $r=b$ at the throat. Eq. (42) for the maximum extraction efficiency corresponds to eq. (4.26) of [23]. The bound (19]) coincides with (4.11) of [23]. In a similar way, there is agreement in other formulas.

It is seen from (172) that simultaneously

$$
\begin{aligned}
& \lim _{b \rightarrow 0} \omega(b, a)=\infty, \\
& \lim _{r \rightarrow \infty} \omega(r, a)=0,
\end{aligned}
$$

so rapid rotaiton in the region $r \sim b$ is compatible with the asymptotic flatness!

\section{SCENARIO 3: COLLISION OF DIFFERENT PARTICLES WITH FINITE ANGULAR MOMENTA OF DEBRIS}

In this Section, we relax restriction (11). We also assume that

$$
L_{3}=O(1), L_{4}=O(1)
$$

and do not fix the relation between $\sigma_{1}$ and $\sigma_{2}$ beforehand. The scenario under consideration does not coincide with previous two cases where $L_{3}$ was big for big $\omega$ according to (49). To avoid cumbersome expressions we consider at once large values of $\omega$ which we are interested in. To ensure the conditions (8) for particles 1 and 2, the angular momenta of initial particles should satisfy

$$
L_{1}<0, L_{2}<0
$$

The case when one of angular momenta is equal to zero is also possible but for simplicity we restrict ourselves by (176) only.

Then, for $\omega \rightarrow \infty$, it follows from (7), that

$$
Z_{i} \approx X_{i}-\frac{N^{2}}{2 X_{i}}\left(m_{i}^{2}+\frac{L_{i}^{2}}{g_{\phi}}\right)
$$

for each particle, $X$ is given by (44). 
We expect

$$
E_{3}=\omega L_{0}+O(1)
$$

where the unknown quantity $L_{0}=O(1)$. The conservation of the radial momentum (23) gives us in the main approximation with respect to $\omega$, when only the leading terms are retained in (77) that

$$
-\sigma_{1} L_{1}-\sigma_{2} L_{2}=L_{0}-L_{3}+\sigma_{4}\left(-L_{0}-L_{4}\right)
$$

where we took into account that $\sigma_{3}=+1$.

The forward-in-time condition (8) for particles 3 and 4 gives rise to

$$
L_{3}<L_{0}<-L_{4}
$$

We must consider different variants separately, depending on signs of radial momenta in the point of collision (further, the sign of $\sigma_{4}$ can change if particle 4 bounces from the turning point). Let us denote corresponding configurations as $\left(\sigma_{1}, \sigma_{2}, \sigma_{4}\right)$. Then direct inspection with (21), (176) and (80) taken into account shows that such configurations are possible: $(+,+;+),(+,-,-)$ and $(-,+,-)$. In the first case eq. (79) turns into identity, so for finding $L_{0}$ one should take into account the corrections in in the expansion (77). In the second case, one finds that $L_{0}=L_{3}-L_{1}$ and in the third one $L_{0}=L_{3}-L_{2}$. By contrast with two previous scenarios, in the second and third cases $E_{3}$ has the same order $\omega$ as $E_{c . m .}$.

\section{DISCUSSION AND CONCLUSIONS}

We generalized observations concerning the properties of the collisional Penrose process that were made in [23] for the Teo wormhole. We revealed the underlying reason of large unbound $E$ (the super-Penrose process) and demonstrated that this happens due to large $\omega$ in the point of collision and has a universal character. Thus we, in a sense, filled a gap in logical classification of the cases of the super-Penrose process and, in addition to the previously considered in literature types of scenarios with small $N$ and large negative $L$, explored also possibilities which are open due to large $\omega$. Thus in combination with previous works [20], [22] all potential factors of unbound $E_{c . m}$. are examined in the "triad" $(N, L, \omega)$. Although we implied that the spacetime approaches the Minkowskian one at infinity, so that the notion of the Killing energy is defined unambiguously, it is clear from derivation that our 
approach admits, in principle, extension to more general asymptotic behavior of the metric as well.

It is worth noting that the results apply to the region where $\omega$ is big irrespective of whether or not the metric is asymptotically flat. Meanwhile, both conditions ( $\omega$ is big inside the ergosphere but $\omega \rightarrow 0$ at infinity) are compatible with each other as it was demonstrated for the Teo wormhole.

It was noticed in [23] that the center-of mass energy of colliding particles is not related directly to the energy extraction. Now, we saw that, moreover, an interesting feature consists in that there exist scenarios in which $E_{3} \gg E_{c . m}$. In particular, high $E$ are consistent with modest $E_{c, m \text {. }}$ for $L_{1}=L_{2}=0$ that generalizes the corresponding scenario for the Teo wormhole [23]. Meanwhile, the aforementioned inequality can be valid in a more general case of nonzero $L_{1,2}$. In another type of scenario, one of angular momenta of outgoing particles is taken to be finite by assumption. Then, $E_{3}=O\left(E_{c . m .}\right)$ is large.

Thus it seems that the super-Penrose process is quite typical for rapidly rotating spacetimes although its details can be different for different scenarios.

As far as the set of objects to which our results can apply is concerned, one can call, in the first place, rotating wormholes. In recent years, interest to such spacetimes increased. Some general properties of slow rotating wormholes were studied in [24], [25]. Exact solutions were obtained in [26] with the use of both analytical and numerical methods. Rotating wormholes were also investigated analytically in [27] (although asymptotically their metric does not have the Minkowskian form). Our results show that nontrivial effects due to particle collisions occur not only for the Teo wormhole [23] but for any kind of a wormhole provided it rotates sufficiently rapidly. The question about potential applications to relativistic stars is less clear. Formally, the results of the present work are valid for them as well. The problem, however, is that for known stars it is difficult to satisfy condition (45).

Meanwhile, we would like to stress that our goal consisted not in the investigation of some concrete properties of particular astrophysically relevant models. Instead, we tried to reveal all potential possibilities of the super-Penrose process as a general phenomenon in gravitation and fill some gaps left after studies in physics of black holes [17], [19], [20], [28], [29] and naked singularities [21]. Our results have a model-independent character and, in this sense, show some interesting general properties of spacetime in the context related to particle collisions. It is the generality of the results that is their main advantage. What is 
especially interesting is that relation between the energy in the centre of mass frame and that measured at infinity can be quite different (that generalizes the corresponding observation made in [23] about the Teo wormhole).

Nonetheless, careful examination of different star models is also of interest that requires, however, a separate treatment.

\section{Acknowledgments}

This work was funded by the subsidy allocated to Kazan Federal University for the state assignment in the sphere of scientific activities.

\section{APPENDIX}

Here, we prove inequality (35). As $L_{1} \leq 0, K \geq K_{+}$where $K_{+}$corresponds to the maximum possible value of $E_{3}=E_{+}+s$. Therefore, it is sufficient to show that $K_{+}>0$. Using (33), (15), (39) one finds

$$
K_{+}=a_{0}+a_{1} L_{1}+a_{2} L_{2}^{2}
$$

where

$$
\begin{gathered}
a_{0}=\omega E_{1}^{2}>0, \\
a_{1}=\frac{N^{2} E_{1}}{g_{\phi}}\left(1+\frac{\omega \sqrt{g_{\phi} B}}{N}\right)-2 E_{1} \omega^{2} . \\
a_{2}=\frac{g_{00}}{g_{\phi}}\left(\omega-\frac{N \sqrt{B}}{\sqrt{g_{\phi}}}\right) .
\end{gathered}
$$

By assumpion, the point of collision is located inside the ergoregion, where (29) is satisfied, hence

$$
\omega \sqrt{g_{\phi}}>N
$$

It is seen from (57) that $B<1$. Then,

$$
a_{2}>\frac{g_{00}}{g_{\phi}}\left(\omega-\frac{N}{\sqrt{g_{\phi}}}\right)>0 .
$$

The coefficent $a_{1}$ can be rewritten as

$$
a_{1}=E_{1} c
$$


where

$$
c=\frac{N^{2}}{g_{\phi}}-2 \omega^{2}+\frac{N}{g_{\phi}} \omega \sqrt{g_{\phi} B} .
$$

Using the inequality $B<1$ again,

$$
c \leq \frac{N^{2}}{g_{\phi}}-2 \omega^{2}+\frac{N}{\sqrt{g_{\phi}}} \omega .
$$

It follows from (85) that

$$
c \leq \frac{N^{2}}{g_{\phi}}-\omega^{2}=-\frac{g_{00}}{g_{\phi}}<0 .
$$

Thus, since $L_{1} \leq 0$, all terms in (81) are positive or, at least, non-negative. This completes the proof.

[1] M. Bañados, J. Silk and S.M. West, Phys. Rev. Lett. 103 (2009) 111102 arXiv:0909.0169.

[2] T. Piran, J. Katz, and J. Shaham, Astrophys. J. 196, L107 (1975).

[3] T. Piran and J. Shaham, Astrophys. J. 214, 268 (1977).

[4] T. Piran and J. Shanam, Phys. Rev. D 16, 1615 (1977).

[5] A. A. Grib and Yu. V. Pavlov, Europhys. Lett. 101, 20004 (2013) arXiv:1301.0698.

[6] O. B. Zaslavskii, Mod. Phys. Lett. A. Vol. 28, 1350037 (2013) arXiv:1301.4699.

[7] N. Tsukamoto and C. Bambi, Phys. Rev. D 91, 084013 (2015) arXiv:1411.5778.

[8] E. Teo, Phys. Rev. D 58, 024014 (1998) arXiv:gr-qc/9803098.

[9] O. B. Zaslavskii, Mod. Phys. Lett. A, 31, 1650029 (2016) [1506.02638].

[10] E. Berti, R. Brito and V. Cardoso, Phys. Rev. Lett. 114, 251103 (2015) arXiv:1410.8534.

[11] O. Zaslavskii, Pis'ma ZhETF 92, 635 (2010) (JETP Letters 92, 571 (2010)), arXiv:1007.4598.

[12] S. Parthasarathy, S.M. Wagh, S.V. Dhurandhar and N. Dadhich, Astrophys. J. 307, 38 (1986).

[13] M. Bejger, T. Piran, M. Abramowicz, and F. Håkanson, Phys. Rev. Lett. 109 (2012) 121101 arXiv:1205.4350.

[14] T. Harada, H. Nemoto and U. Miyamoto, Phys. Rev. D 86 (2012) 024027 [Erratum ibid. D 86 (2012) 069902] arXiv:1205.7088.

[15] O. Zaslavskii, Phys. Rev. D 86 (2012) 084030 arXiv:1205.4410].

[16] J. D. Schnittman, Phys.Rev.Lett. 113, 261102 (2014), arXiv:1410.6446.

[17] E. Leiderschneider and T. Piran, arXiv:1510.06764].

[18] O. Zaslavskii, Mod. Phys. Lett. A 30 (2015) 1550076, arXiv:1411.0267. 
[19] E. Leiderschneider and T. Piran, arXiv:1501.01984].

[20] O. B. Zaslavskii, Europhys. Lett. 111 (2015) 50004, arXiv:1506.06527].

[21] M. Patil and T. Harada, arXiv:1510.08205].

[22] O. B. Zaslavskii, arXiv:1510.02140].

[23] N. Tsukamoto and C. Bambi, Phys. Rev. D 91, 104040 (2015) arXiv:1503.06386].

[24] P.E. Kashargin, S.V. Sushkov, Grav.Cosmol.14, 802008 arXiv:0710.5656.

[25] P.E. Kashargin, S.V. Sushkov, Phys.Rev.D 78, 064071,2008 arXiv:0809.1923].

[26] B. Kleihaus and J. Kunz, Phys. Rev. D 90, 121503(R) (2014) arXiv:1409.1503.

[27] K. A. Bronnikov, V. G. Krechet, J. P.S. Lemos, Phys. Rev. D 87, 084060 (2013) arXiv:1303.2993.

[28] K. Ogasawara, T. Harada, and U. Miyamoto arXiv:1511.0011.

[29] O. B. Zaslavskii, Phys. Rev. D 93, 024056 (2016) arXiv:1511.07501.

[30] O. B. Zaslavskii, Phys. Rev. D 84, 024007 (2011) arXiv:1104.4802.

[31] J. M. Bardeen, W. H. Press, and S. A. Teukolsky, Astrophys. J. 178, 347 (1972).

[32] L. Smarr. Phys. Rev. Lett. 30, 71 (1973).

[33] O. B. Zaslavskii, Phys. Rev. D 92, 044017 (2015) arXiv:1506.00148. 